\title{
Body Area Networks
}

\author{
G. Prabhakara Reddy**, P. Bhaskara Reddy*, V. Koti Reddy** \\ * Department of Electronics and Computer Engineering, MLRIT, Hyderabad \\ ** Department of Computer Science \& Engineering, MLRITM, Hyderabad
}

\begin{abstract}
Recent technological advances in integrated circuits, wireless networks, and physiological sensing have enabled miniature, lightweight, low power, intelligent monitoring devices to be integrated into a Body Area Network (BAN). This new type of technology hold much promise for future patient health monitoring. BANs promise inexpensive, unobtrusive, and unsupervised ambulatory monitoring during normal daily activities for long periods of time. However, in order for BANs to become ubiquitous and affordable, a number of challenging issues must be resolved, such as integration, standardisation, system design, customisation, security and privacy, and social issues. This paper presents an overview of many of these issues and indeed the background and rationale of body area networks.
\end{abstract}

Keywords: body area networks, wireless networks, physiological sensing, micro-technology

\section{Introduction}

A Body Area Network (BAN) is a collection of advanced nano and micro-technology components. They are designed to improve the speed and accuracy of how data is recorded. In general a Body Area Network has sensors and actuators that are used to monitor and log data, this data is then transmitted and stored to a base station. When the data has reached the base station it is possible for it to be sent on to devices via the internet for others to access. Body Area Networks are a relatively modern invention and are primarily being designed for use within the health industry. Their main purpose is to enable doctors and other medical staff to safely monitor the health status of patients. One of the benefits of Body Area Networks is that those patients with chronic diseases such as Alzheimer's, Asthma and Diabetes can be monitored much more closely. Doctors will have the power to update patient records quickly and efficiently and they will be able to store information on the patients' general health.

Hospitals throughout the United States and in Europe have been running pilot programmes to experiment with monitoring patients' health. They have introduced ID bracelet's that contain chips and antennas, which can follow the patients' whereabouts. The system will also use antennas to track individuals as they walk about the hospital and send alerts if a patient begins to collapse. A company called Zarlink are currently working on a project named 'Healthy Aims' which is focusing on in-body devices that will help millions of people world-wide. Body Area Networks take advantage of the low power radio frequencies (RF) and enable the Body Area Network to supply the patients' data in real time. There is a frequency range dedicated to Body Area Networks, known as MICS (Medical Implantable Communication Service) band, it operates between 402 and $405 \mathrm{MHz}$ and is specifically for implanted devices to communicate with other external devices.

Not only will doctors be able to monitor patients' general health but they will also have the ability to change the settings for specific implanted devices so that they perform much better, thus improving the patients health. Currently individuals requiring pacemakers have to endure the pain and stress of surgery in order to have their pacemaker device fitted. The very first implantable pacemaker invented was produced in the 1960's and has evolved tremendously since. The pacemakers set up as part of a Body Area Network wirelessly send the patients' health status to a near-by RF transceiver. From this RF transceiver the data is transmitted to a doctor. The fact that the patients health status is regularly being forwarded to the doctor means that their health record is always up-to-date and the information reaches the doctor in real-time. If at any stage in life a patient encounters problems with their current pacemaker then it is quite possible that they will have to endure more surgery simply to alter the 
settings on the pacemaker. Therefore the added benefit of the 'Healthy Aims' project is the fact that the patient will only ever need to have surgery performed once and this is only to have the device fitted. Doctors and medical staff will be able to monitor the health status of many patients without their need to visit the clinic. Doctors can stay in their office and work their way through the patients' records; if they discover that a patients' health status is below normal they will be able to prepare a prescription or make an adjustment without the patient being aware. The doctor will then simply have to contact the patient to inform them of the outcome. The doctor is able to do this because of the fact that the patient Body Area Network independently transmits data to the doctor, therefore crisis information will reach the doctor much quicker than if somebody discovered the patient unconscious on their bathroom floor and made an emergency call to the ambulance service.

Having a Body Area Network in place would mean that individuals would not have to travel to the clinic, which means that there would be less pollution made to the environment. Also, the clinic will then have fewer visiting patients to check, which will mean that the waiting times for those patients in the waiting area would be reduced immensely. This complex method of communication between the patient and the doctor is made possible by a two-way RF link. The sensors of a Body Area Network are extremely compact and complex in design. The fact that the sensors are so minute means that the patients will be able to lead a normal life, as the sensor devices are very unobtrusive. All sensors produced will contain the same basic elements such as a power supply and wireless transceiver as well as a control mechanism, a sensor and the casing that will hold all of the components together. The sensors will be designed in a way that allows them to be self-governing for the entire lifetime. These sensors are designed to "measure and monitor events and physical properties such as temperature, movement, pressure, and location.

BAN's work through a process of data being transmitted from an implanted device to an external device. The sensor that is implanted inside the patient's body wirelessly interacts with other sensors and actuators. An actuator is the mechanism by which an agent acts upon an environment. The agent can be either an artificial intelligent agent or any other autonomous being. An autonomous being can be a human being or an animal. The Body Area Network functions by passing data from each sensor to a main station. The main station then fuses the data passed from each of the sensors and it is then sent to a recipient via the internet. The IMEC (Interuniversity Micro Electronics Center) has stated that "At the system level, it is concentrating on wireless communications in and around the human body; ad-hoc networking of wireless nodes; reliability, accuracy and sensitivity issues; position determination; and design technology for a fast reproducible design cycle. Ad hoc networking is the best suited networking type for Body Area Networks because of the fact that it is comprised of small wireless electronic devices. The IMEC is working on implementing the ability to monitor the location of the patients within the hospital. This means that the patients will have the freedom to walk around the hospital or go outside for fresh air; the sensors within the Body Area Network will be programmed to detect the patients' temperature and other vital signs. Therefore, if a patient suddenly collapses the doctors and medical staff will be notified; and can rush to the patient's aid and provide treatment. Next we examine in more detail the field of wireless body area networks.

\section{Body Area Networks}

A BAN consists of a collection of small sensor nodes that are placed around the body. These may be attached directly to the skin or as part of special clothing. Each node has either a small power source or takes power from the body. The nodes can collectively communicate with a central node (like a PDA) which can in turn connect to the internet and in doing so can relay the data from the sensors to a particular application or person. There should be no wires involved, making the BAN as unobtrusive as possible as illustrated in Figure 1. 


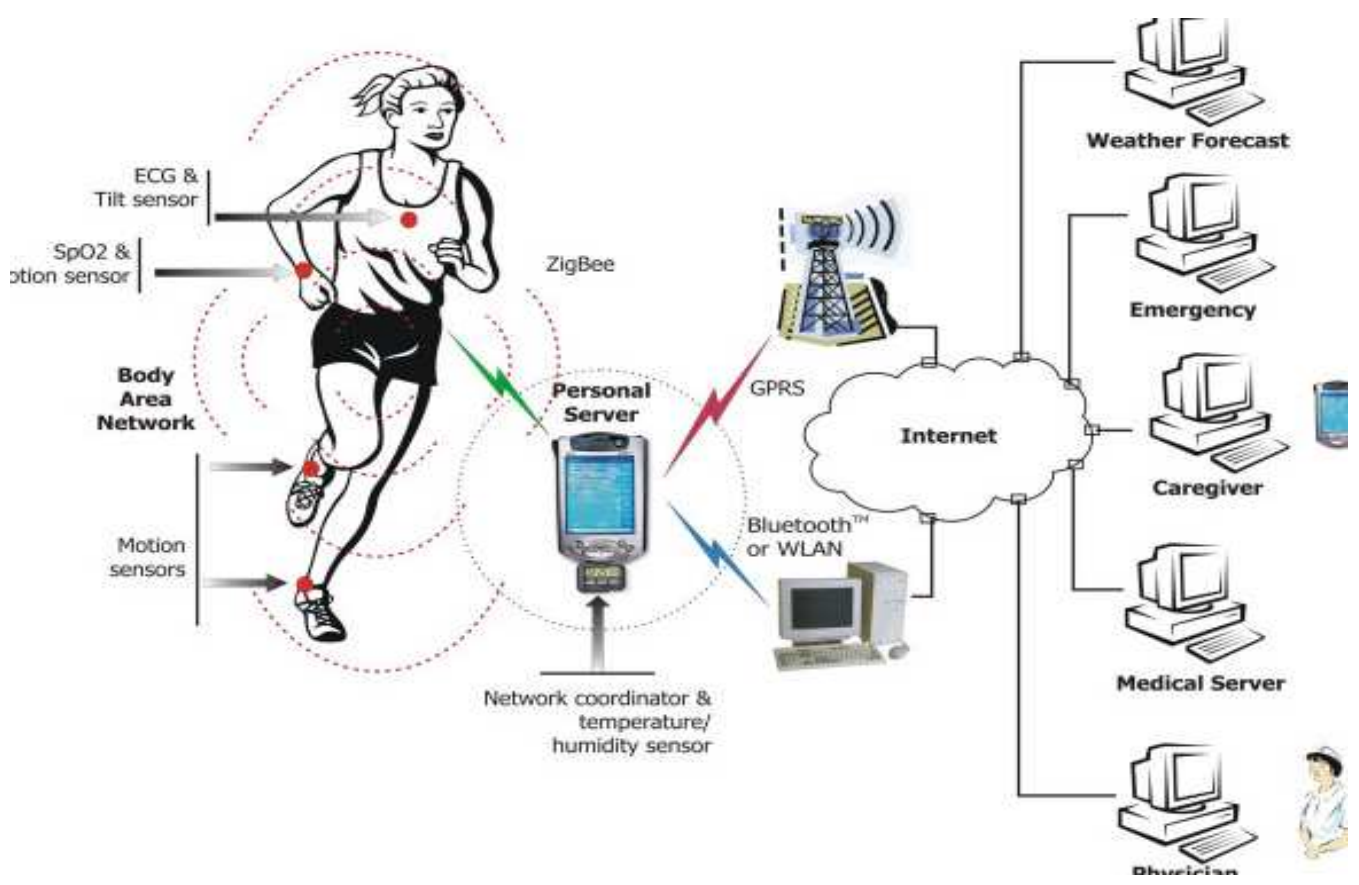

Figure 1. How a Body Area Network is linked to the Internet (Jovanov, 2005)

To engineer a successful BAN, a number of challenges exist which must be overcome. New methods need to be developed to address the following such as:

- The lifetime of the power sources in the sensors needs to be increased and/or methods to get energy from other sources (e.g.: body heat) need to be perfected.

- The interaction of the sensors (nodes) and the main node (PDA) needs to be designed in such a way so as to allow specialist applications to be written to do particular functions (e.g.: disease management).

- The device needs intelligence (memory and processing capabilities) to allow it to process, transfer and store data.

- Chemical, Physical and Biological measurement needs to be integrated with the sensors to give a clear picture of human physiology and the BAN components need to be integrated in a reliable and cost effective way.

\subsection{History of BANs}

The development of the PAN (Personal Area Network) grew out of the work being done by a number of different groups working in MIT (Massachusetts Institute of Technology) on the 90's. The original groups wanted to interconnect information appliances that were carried on the body. Also they wanted to measure positioning using electric field sensing and they realised that they could send data through the body by modulating the electric field. Thomas G. Zimmerman was one of the main players in the development of the PANs. He developed technology that has the effect of allowing the body to act like a copper cable, basically like a conductor. The other main player in those early days was Neil Gershenfeld who was in charge of the Physics and Media Group in MIT. His group applied a method known as "near-field coupling" to particular problems. This allows them to determine the accurate position of one part of the body in relation to another. They placed pairs of antennas on parts of the body (e.g.: on the hand and elbow). By running an electric current between them they noted that as the parts moved (i.e. flex of the elbow) the capacitance of the circuit was changed. By measuring the capacitance they were able to determine the positions of the antenna.

Zimmerman solved a problem they encountered. If a hand was placed between the antennas then the measurement was no longer accurate. He was able to show that some of the current was passing through the body, therefore affecting the measurement. Another group working at the media lab then asked these two to develop a network to connect together all the 
electric gadgets that a person might carry on their person. Many people carried around a number of digital devices but none of them could communicate with each other. A person might have a mobile phone, a pager, a PDA and a digital watch all about their person at the same time. This person might receive a page, then type the number into the PDA to see whose it is, then dial it on the mobile phone. Too much effort is being wasted on a very low-level information exchange. Zimmerman and Gershenfeld noticed that if they modulated the electric field flowing through a person's body, they could make it represent a 1 or 0 , thus allowing the body to carry digital information. The discovered that if the frequency and power used were both kept very low then the signal would not propagate far beyond the body. This would mean that only devices on the body or in direct contact with it could detect the signal. The current used is very small and is totally unnoticed by the person. These ideas of how a PAN developed were the early attempts at creating a BAN.

\subsection{Applications of BANs}

Among the many possible applications that have been thought up for BANs are communication in hospitals, communication on aeroplanes or spaceships, monitoring of patients at home (post operative care), modern Warfare, monitoring of babies, interlinking of components in home entertainment products and athlete monitoring and sports analysis

Medical Applications

BANs can provide interfaces for diagnostics, for remote monitoring of human physiological data, for administration of drugs in hospitals and as an aid to rehabilitation. In the future it will be possible to monitor patients continuously and give the necessary medication whether they are at home, in a hospital or elsewhere. Patients will no longer need to be connected to large machines in order to be monitored.

\section{Sports Applications}

In the sporting arena is will be possible to take many different readings from an athlete without having them on a treadmill in a laboratory. The ability to measure various levels during real life competition, a race for example, would give coaches a more accurate picture of their athlete's strengths and weaknesses. Another application of using BANs in sport to monitor athletes closely.

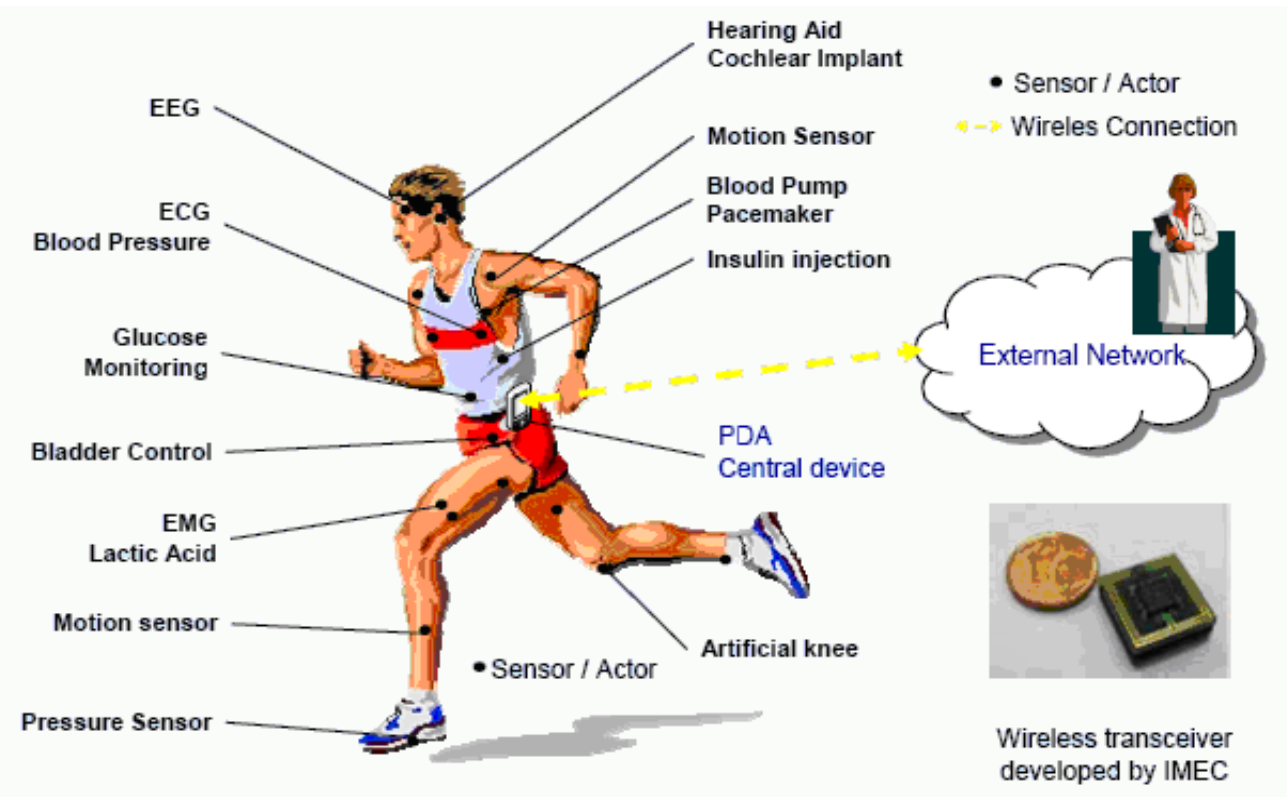

Figure 4. A BAN on an Athlete (Latré, 2005)

\section{Military Applications}

The opportunities for using BANs in the military are numerous. Some of the military applications for BANs include monitoring health, location, temperature and hydration levels. 
These readings can then be used to administer Casualty care (e.g.: morphine), knowledge of when to enhance strength, concentration, accuracy (e.g.: drugs) and possibly to reduce friendly fire incidents (tell them who each other is and where).

\subsection{Sensors and Actuators}

In a BAN two main types of non-computer device can be identified known as sensors and actuators. The Sensors measure certain human body parameters such as temperature, heart rate, EEG, pressure on joints, etc. These measurements can be taken inside or outside of the human body. Actuators take particular actions depending on the information received by the sensors and instructions given via the PDA and the web. Administration of insulin for diabetics would be an example of something that the actuators could control. (Jovanov, 2005) lists some of the specific sensors that could be used in their version of a wireless BAN such as:

- An ECG (electrocardiogram) sensor for monitoring heart activity.

- An EMG (electromyography) sensor for monitoring muscle activity.

- An EEG (electroencephalography?) sensor for monitoring brain electrical activity.

- A blood pressure sensor.

- A tilt sensor for monitoring trunk position.

- A breathing sensor for monitoring respiration.

- Movement sensors used to estimate a users' activity.

Most of the sensors that are in use are of an electronic or electrical nature. These types of sensor may sense a change in current or resistance in a body (a muscle for example). When the muscle is contracted it would give a different reading to a small voltage applied to it than if it was relaxed. It is these differences that are measured by the sensor. In a very simplistic version a totally relaxed state (i.e.: laying down) might correspond to a value of 0 , but a tensed state (i.e.: standing on one leg) might give a value of 10 . Any readings in between 1 and 10 will correspond to the state of relaxation/contraction of the muscle. When this information is sent back to the PDA and then on to the main computer it would be possible to know at all stages the activity state of a muscle. For instance, if a large number of sensors were placed on the legs of a runner and all the data was recorded over an $800 \mathrm{~m}$ race, it would be possible to determine at exactly what point the runner was getting tired or was losing form. Training could then be tailored specifically; i.e. strengthen calf muscles because the runner becomes flatfooted at $650 \mathrm{~m}$ and therefore slows down.

An actuator is a device that transforms an input signal into motion. If one was used to control the amount of insulin that a diabetic was receiving, then an instruction given by a computer could control the amount of the chemical released into the body. A medical person could send this instruction directly to the BAN that the patient was wearing no matter where they were located; via the Internet, WiFi, Bluetooth or some other means of communicating the signal to the PDA, which in turn would control the actuator. Along with the sensors, the actuators are engineering components, which allow the human body to be influenced, monitored or controlled by the computer system.

\section{$2.4 \quad$ Networking Body Area Networks}

When creating a BAN, a number networking issues need to be addressed. Direct communication is where a node sends data directly to a central device. If a node were on the foot, its data would have to pass other nodes on the way to the PDA meaning that a number of data pathways would exist in the same tissue at the same time. This could cause interference and could also cause unnecessary temperature rise in the tissue however with Multihop communication, data is sent through the intermediate nodes on the way to its destination. Each of the nodes acts as a small router. This would eliminate internal interference. This is also more energy efficient than the direct route.

IEEE 802.15 oversees the standards for how Wireless PANs communicate. These standards are essential to ensure that all of the different groups who are involved in designing the BANs make sure that everything will work with everything else. The 802.15 standard has 4 task groups, each of which has authority over the development of a certain type of technology which will be used in BANs such as WPAN/Bluetooth, WPAN/ Wireless Local Area Networks, WPAN High Rate (20Mbit/s or higher) and WPAN Low Rate (long battery life - Zigbee). They are at different stages of development and will eventually ensure that BANs created in one country will work with those in another. 


\subsection{Software}

One of the main options that is available and in use (and development) is "Tiny OS" which was developed by UC Berkley in California. This is an open source operating system for wireless embedded sensor networks. It is an extremely small OS (Operating System) in terms of code and memory resources. This is what makes it suitable for sensor networks where memory is at a minimum. A lot of the groups that are currently using Tiny OS are involved in research using sensor networks to monitor some phenomenon or other. Some examples include sensor networks to monitor volcanic eruptions in Ecuador and tracking of fire fighters in buildings.

Another option in control software is to use MSR Networked Embedded Sensing Toolkit (MSR Sense) from Microsoft. This is a collection of software tools that allow users to collect, process, archive and visualise data from a sensor network Other options are available and in development so it remains to be seen which will come to the fore. At the moment Tiny OS and MSR are freely available to download.

\section{Social Issues}

A number of issues exist regarding the creation of BANs include system design issues and human issues. System Design Issues include (Jovanov, 2005):

\section{Sensor Types}

What type of sensor should be included in the BAN? This will depend on where it is to be used and for what purpose

\section{Power Sources}

If the BAN is designed to be used for a long period of time then the power sources must be appropriate. If it is going to be used for some short intense activity then a different source could be used.

\section{Wireless Communication Range}

Is the person using the BAN likely to remain within a particular area? e.g.: a hospital, or are they likely to be outdoors? E.g.: a soldier in the desert.

4. Sensor location and mounting

Could they be woven into the uniform of a solider or might they need to be small unobtrusive implants in the skin?

\section{Weight and size of sensor}

If the person is confined to bed at home then the sensors could be of a different type from those used on a runner.

6. Coexistence Issues

Will the system interfere with other systems that are in place such as a pacemaker or the hearing aid of a patient.

\subsection{Issues Arising from BAN Trials}

A number of major trials are being conducted all over the world such as the MobiHealth trial (Bults, 2004) which is being carried out in four European countries. MobiHealth was used by medical personnel to monitor a range of conditions in patients outside of hospitals. The 9 trials that were carried to date were:

1. Germany: Telemonitoring of patients with cardiac arrhythmia.

2. The Netherlands: Integrated homecare for women with high-risk pregnancies.

3. The Netherlands: Tele trauma team

4. Spain: Support of home based healthcare services.

5. Spain: Outdoor patient rehabilitation.

6. Sweden: Lighthouse alarm and locator trial.

7. Sweden: Physical activity and impediments to activity for women with RA.

8. Sweden: Monitoring the vital parameters in patients with respiratory insuffiency.

9. Sweden: Homecare and remote consultation for recently released patients in a rural area. 


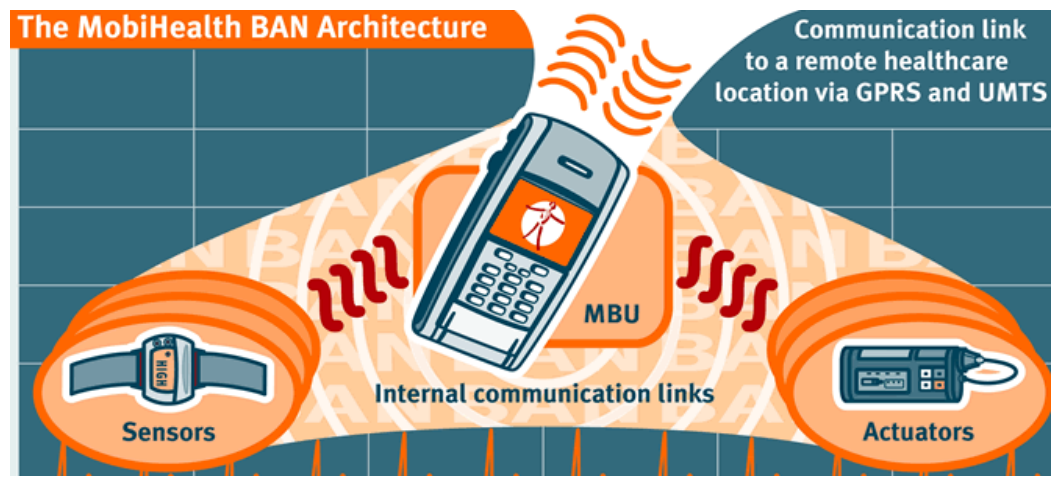

Figure 2. Mobile Health BAN Architecture [Source: http://www.mobihealth.org/]

As a result of these trials a number issues arose such as data rates fluctuating, operator errors, handover errors (e.g.: changing from one network to another), bandwidth issues, IP address allocation and power supplies insufficient.

Other issues regarding BANs in general include privacy \& security, legal issues and control issues. For instance, will the data that is being transferred remain private? Is it secure and would it be possible for someone to send false information to a BAN and tell it to perform some dangerous function? e.g.: make you warmer or make you overdose. Is it legal to hold all information about a person in case a company could detect from records that a person had a weak heart. Finally, in an advanced BAN important functions might be controlled by a computer such as the ability to fall asleep or stay awake being controlled by an actuator that regulated the flow of a certain chemical in the body. All of these issues will need to be addressed and before Body Area Networks become a part of everyday life.

\section{Conclusion}

A major issue with Body Area Networks is security. As data that has been logged is transmitted over the internet there is always the possibility of someone else hacking into it. Therefore, in order to prevent a hacker from gaining access to the network and corrupting the data, it is vital that there is adequate encryption in place. In order to ensure that it is impossible for anyone to hack into a Body Area Network thorough testing must be carried out. Also, because each patient is unique and requires different treatments the data held on one patient will be completely different to the data held on another patient. Therefore, it is extremely important that each patients individual Body Area Network is not confused with one another. The use of chips that can wirelessly communicate via a Body Area Network is an excellent idea, but only if they are used in an ethical manner. Body Area Networking can be used to allow an individuals personal details to be stored on a chip, which can then easily interact with other devices.

\section{References}

[1] Jovanov E, Milenkovic A, Otto C and de Groen P. A wireless body area network of intelligent motion sensors for computer assisted physical rehabilitation. Journal of Neuroengineering Rehabilitation. 2005; 2: 6. 2005 March 1. doi: 10.1186/1743-0003-2-6.

[2] Latré B, Moerman I, Dhoedt B, Demeester P. (2005) Networking in Wireless Body Area Networks, http://www.ibcn.intec.ugent.be/css design/research/topics/2005/Networking\%20in\%20Wireless\%20B ody\%20Area\%20Networks.pdf

[3] Bults R, Wac K, Van Halteren A, Konstantas D, Jones V, Widya I. Body Area Networks for Ambulant Patient Monitoring Over Next Generation Public Wireless Networks. Prroceedings of the 3th IST Mobile and Wireless Communications Summit 27-30 June 2004, Lyon, France.

JTI Vol. 1, No. 1, March 2013: $36-42$ 